\title{
Novidades TAXONÔMICAS EM ESPÉCIES BRASILEIRAS DE CROTALARIA Sect. Calycinae Wight \&Arn. (Leguminosae-Papilionoideae)
}

\author{
Andréia Silva Flores ${ }^{1,2}$, Andrea Martinelli Filliettaz ${ }^{1}$ \\ \& Ana Maria Goulart de Azevedo Tozzi $i^{2,3}$
}

\section{Resumo}

(Novidades taxonômicas em espécies brasileiras de Crotalaria sect. Calycinae Wight \& Arn. (LeguminosaePapilionoideae)) São propostas as sinonimizações de quatro espécies e de duas variedades: Crotalaria brasiliensis Windler \& S.G. Skinner em Crotalaria grandiflora Benth., Crotalaria barretoensis Windler \& S.G.Skinner em Crotalaria martiana Benth. subsp. martiana, Crotalaria hatschbachii Windler \& S.G.Skinner, Crotalaria hatschbachii var. sericea Windler \& S.G.Skinner, Crotalaria bellii Windler \& S.G. Skinner e Crotalaria flavicoma var. major Micheli em Crotalaria martiana subsp. mohlenbrockii (Windler \& S.G.Skinner) Planchuelo. Também são propostos a transferência de $C$. paraguariensis da sinonímia de $C$. martiana subsp. martiana para C. martiana subsp. mohlenbrockii e o restabelecimento de $C$. subdecurrens Mart. ex Benth. à categoria específica.

Palavras-chave: Crotalaria, Calycinae, Leguminosae, Brasil, taxonomia.

\section{Abstract}

(Taxonomic novelties in Brazilian species of Crotalaria sect. Calycinae Wight \& Arn. (LeguminosaePapilionoideae)) The synonymy of four species and two varieties: Crotalaria brasiliensis Windler \& S.G. Skinner to Crotalaria grandiflora Benth., Crotalaria barretoensis Windler \& S.G.Skinner to Crotalaria martiana Benth. subsp. martiana, Crotalaria hatschbachii Windler \& S.G.Skinner, Crotalaria hatschbachii var. sericea Windler \& S.G.Skinner, Crotalaria bellii Windler \& S.G. Skinner and Crotalaria flavicoma var. major Micheli to Crotalaria martiana subsp. mohlenbrockii (Windler \& S.G.Skinner) Planchuelo is proposed. Also, the transference of C.paraguariensis from synonymy of C. martiana subsp. martiana to C. martiana subsp. mohlenbrockii and the reinstatement of $C$. subdecurrens Mart. ex Benth. to the specific rank are proposed.

Key-words: Crotalaria, Calycinae, Leguminosae, Brazil, taxonomy.

\section{INTRODUÇão}

As espécies de Crotalaria são caracterizadas por apresentarem folhas digitadotrifolioladas, unifolioladas ou simples, androceu monadelfo aberto na base, anteras dimorfas e legumes inflados. O gênero é considerado o terceiro maior de Papilionoideae, com cerca de 600 espécies distribuídas pelos trópicos e subtrópicos, com a maioria ocorrendona África tropical, considerada como o centro de diversidade das espécies (Polhill 1981).

A classificação infragenérica atual de Crotalaria foi estabelecida por Polhill (1968) e reformulada por Bisby \& Polhill (1973), a partir de caracteres morfológicos florais, como a forma do receptáculo, grau de torção do bico da quilha, posição dos apêndices no estandarte, cálice bilabiado ou não e a forma do estilete. As espécies do gênero estão agrupadas em oito seções, sendo que quatro estão representadas no Brasil: duas destas apresentam elementos nativos: Crotalaria sect. Calycinae e Crotalaria sect. Chrysocalycinae (Benth.) Bak.f. enquanto que Crotalaria sect. Hedriocarpae Wight \& Arn. e Crotalaria sect. Crotalaria apresentam espécies exóticas.

As espécies pertencentes à seção Calycinae caracterizam-se principalmente por apresentar o ápice das peças da quilha torcido, apêndices restritos à lâmina do estandarte $\mathrm{e}$ cálice profundamente bilabiado, geralmente tão longo quanto a corola. A seção possui cerca de 70 espécies, principalmente na Ásia, estendendo também na Austrália, África e na região Neotropical.

Na América do Norte, parte das espécies desta seção foi tratada por Rafinesque (1836), denominando-a como subgênero Iocaulon, e

Artigo recebido em 07/2005. Aceito para publicação em 10/2005.

${ }^{1}$ Programa de Pós-Graduação em Biologia Vegetal - Unicamp

${ }^{2}$ Universidade Estadual de Campinas, Instituto de Biologia, Departamento de Botânica, CP 6109, CEP 13083-970, Campinas, São Paulo, Brasil.

${ }^{3}$ Autor para correspondência: anatozzi@unicamp.br 
por Windler (1971). Para o Brasil, as espécies de Calycinae foram estudadas por Bentham (1859) na série Simplicifoliae, então constituída por 21 espécies, por Filliettaz (2002), que reconheceu 14 espécies nativas e Flores (2004), que tratou as espécies de Crotalaria pertencentes às quatro seções ocorrentes no Brasil.

Como resultado destes dois últimos trabalhos (Filliettaz 2002, Flores 2004), algumas alterações na circunscrição de determinados táxons foram propostas e o objetivo do presente artigo é formalizar as delimitações taxonômicas aceitas para as espécies de Crotalaria sect. Calycinae ocorrentes no Brasil.

\section{Material e Métodos}

Os resultados deste trabalho basearamse nos dados do tratamento taxonômico do gênero Crotalaria no Brasil (Filliettaz 2002, Flores 2004). Os dados foram obtidos através da análise de exsicatas, fotografias e coleções tipo dos seguintes herbários: BHMH, C, F, HB, IAN, K, M, MBM, MO, NY, R, RB, SP, UEC (siglas conforme Holmgren et al. 1990).

\section{Resultados e Discussão}

1. Crotalaria grandiflora Benth., Ann. Nat. Hist. 3: 429. 1839.

Crotalaria acutiflora var. grandiflora (Benth.) Benth., Fl. bras. 15(1): 23. 1859. Typus: BRASIL. near Cercado, Pohl (holótipo K; foto holótipo F!).

Crotalaria brasiliensis Windler \& S. G. Skinner, Phytologia 50: 194. 1982. Typus: BRASIL. DISTRITO FEDERAL: Cachoeira Piripiripau, ca. $15 \mathrm{~km} \mathrm{~S}$ of Planaltina, H. S. Irwin et al. 26425 (Holótipo MO; isótipo IAN!; foto holótipo MO!) syn. nov.

Crotalaria brasiliensis foi descrita durante a preparação da monografia de Crotalaria para a Flora Neotropica (Windler \& Skinner 1982a), sendo conhecida somente pela coleção tipo e por um outro material coletado no Brasil e depositado no herbário em Paris (P).

Comparando-se a descrição de $C$. brasiliensis (Windler \& Skinner 1982a) com a de C. grandiflora (Bentham 1839) e com os materiais identificados como C. grandiflora, foi observado que as características são muito semelhantes, inclusive nas dimensões das folhas e flores. A sinonimização de C. brasiliensis também foi baseada pela distribuição simpátrica com C. grandiflora, no Distrito Federal (Brasil) e devido ao caráter diagnóstico utilizado pelos autores (Windler \& Skinner 1982a) para diferenciarem estas espécies que foi a posição da inflorescência. Segundo os autores, $C$. brasiliensis possui inflorescência opositifólia enquanto que em $C$. grandiflora a inflorescência é terminal em ramos axilares curtos. No entanto, em C. brasiliensis foi constatada a presença de inflorescências axilares em maior freqüência do que opositifólia.

2. Crotalaria martiana Benth. subsp. martiana, London J. Bot. 2: 482. 1843. Crotalaria foliosa var. martiana (Benth.) Benth., Fl. bras. 15(1): 24. 1859. Crotalaria martiana Benth. subsp. martiana sensu Planchuelo, Candollea 53(2): 462. 1998, excl. syn. Crotalaria paraguayensis Windler \& S. G. Skinner. Typus: BRASIL. C. F. P. Martius 1142 (holótipo M; foto holótipo M!; isótipo NY; foto isótipo NY!)

Crotalaria barretoensis Windler \& S. G.Skinner, Phytologia 50(3): 189. 1982. Typus: BRASIL. MINAS GERAIS. Serra do Cipó, estrada de Conceição, município Conceição, XI.1938, M. Barreto 8602 (holótipo F!; isótipos BHMH!, R!) syn. nov.

A sinonimização de Crotalaria barretoensis em C. martiana subsp. martiana foi baseada nas afinidades morfológicas marcantes, como forma de folha e pétalas da quilha, e de distribuição geográfica. Windler \& Skinner (1982a), ao estabelecerem $C$. barretoensis, caracterizaram-na por apresentar hábito arbustivo, inflorescências terminais e brácteas truladas a ovais, sendo endêmica na Serra do Espinhaço, Minas Gerais. Entretanto, analisando os materiais correspondentes a estes táxons foi verificado que estes não apresentam caracteres constantes para sua distinção. Ambos os táxons possuem o mesmo tipo de hábito arbustivo, as inflorescências podem ser terminais 
ou algumas vezes opositifólias e as brácteas são foliáceas, variando de ovais a truladas.

\section{Crotalaria martiana subsp. mohlenbrockii} (Windler \& S.G.Skinner) Planchuelo, Candollea 53(2): 465. 1998. Crotalaria foliosa Benth., Ann. Nat. Hist. 3: 429. 1839, non Rottler, 1803, Crotalaria mohlenbrockii Windler \& S. G. Skinner, Brittonia34(3): 344. 1982. Crotalaria foliosa var. a obovata Benth., Fl. bras. 15(1): 24. 1859, nom. superfl. Typus: BRASIL. MINAS GERAIS: Montes Claros, Pohl 1122 (lectótipo W, designado por Windler \& Skinner (1982b); isolectótipo K).

Crotalaria paraguayensis Windler \& S.G. Skinner, Brittonia 34(3): 343. 1982. Typus: PARAGUAI. CORDILLERA: Cordillera de Altos, Cerro Choché, IV-1902, Fiebrig 226 (holótipo US; isótipos BAF, BM, F, G, K, W; foto isótipo K!) syn. nov.

Crotalaria hatschbachii Windler \&

S.G Skinner, Phytologia 50(3): 200. 1982. Typus: BRASIL. MATO GROSSO: Rondonópolis: Serra da Petrolina, XII-1974, G. Hatschbach 34139 (holótipo NY; isótipo BALT, C!, HB!, MBM!; foto holótipo NY!) syn. nov.

Crotalaria hatschbachii var. sericea Windler \& S. G Skinner, Phytologia 50(3): 202. 1982. Typus: BRASIL. GOIÁS: Estrada Alto Paraíso-Campo Belo km 41, XII-1976. G. J. Shepherd et al. 3735 (holótipo NY; isótipo UEC!; Foto holótipo NY!) syn. nov.

Crotalaria bellii Windler \& S. G. Skinner, Phytologia 50(3): 62. 1982. Typus: BRASIL. GOIÁS: Chapada dos Veadeiros, ca. $30 \mathrm{~km}$ NW of Veadeiros, II-1996, H. S. Irwin et al. 12951 (holótipo US; isótipos K, MO, NY, RB!, SP!; foto isótipo NY!) syn. nov.

Crotalaria flavicoma var. major Micheli, Vidensk. Meddel. Dansk Naturhist. Foren. Kjøbenhavn 1875: 62. 1875. Typus: BRASIL. SÃO PAULO: São Carlos, in campis. I-1834. Lund (holótipo C!; isótipo C!) syn. nov.

Crotalaria mohlenbrockii é um nome novo proposto por Windler \& Skinner (1982b) para $C$. foliosa Benth., que é um homônimo posterior de $C$. foliosa Rottler.
Crotalaria hatschbachii, Crotalaria hatschbachii var. sericea, Crotalaria bellii e Crotalaria flavicoma var. major são táxons muito relacionados a $C$. martiana subsp. mohlenbrockii, sendo que $C$. hatschbackii foi diferenciada desta apenas por apresentar inflorescência opositifólia. Entretanto, da mesma forma que foi observado para C. martiana, a inflorescência é predominantemente terminal, mas em vários espécimes foi constatada a presença de inflorescências opositifólias. Segundo Windler \& Skinner (1982a) Crotalaria belliié uma espécie restritana região da Chapada dos Veadeiros (Goiás), porém analisando os materiais citados como parátipos pelos autores, observou-se que um destes (Irwin et al. 12673) se tratava de $C$. grandiflora Benth., enquanto nos isótipos analisados foi constatada a semelhança com $C$. martiana subsp. mohlenbrockii, principalmente, em relação à forma das folhas e tamanho de flor.

Através da análise do material tipo, também foi constatado que $C$. flavicoma var. major não apresenta descontinuidades que justifiquem seu reconhecimento como táxon distinto e esteé um exemplar tipicamente pertencente a C. martiana subsp. mohlenbrockii. Considerando $C$. flavicoma, apilosidade hirsuta dos ramose folhas é um caráter diagnóstico que possibilita seu pronto reconhecimento em relação a $C$. martiana subsp. mohlenbrockii que possui pilosidade serícea nestas estruturas.

Windler \& Skinner (1982b) reconheceram três espécies neste complexo: descreveram $C$. paraguayensis, propuseram o nome novo $C$. mohlenbrockii para $C$. foliosa e restabeleceram a categoria de espécie para $C$. martiana Benth. Os autores distinguiram as três espécies principalmente pelo comprimento das inflorescências, tamanho e forma de folhas, pilosidade do cálice e na distribuição geográfica do grupo. Posteriormente, Planchuelo (1998) propôs $C$. martiana como única espécie com duas subespécies baseadas nocomprimento das inflorescências. C. paraguayensis foi sinonimizada a $C$. martiana típica e $C$. mohlenbrockii passou a subespécie de C. martiana. As semelhanças morfológicas e de distribuição 
geográfica entre $C$. paraguayensis e $C$. martiana subsp. mohlenbrockii são mais fundamentadas, de forma que é proposta a transferência deste táxon da sinonímia da subespécie típica para a subespécie mohlenbrockii.

4. Crotalaria subdecurrens Mart. ex Benth., Fl. bras. 15(1): 20. 1859. Typus: BRASIL. "Habitat in provinciae Minarum campis ad Contendas", C.F.P. Martius 1606 (lectótipo M, foto lectótipo M!, designado por Windler \& Skinner, Phytologia 49: 429. 1981).

Crotalaria subdecurrens foi descrita por Bentham (1859) e comparada com $C$. breviflora DC., especialmente quanto à inflorescência e bráctea, e com $C$. grandiflora quanto ao estandarte. Diferenciada de $C$. breviflora pelas flores maiores e de $C$. grandiflora pelas estípulas (Bentham 1859), foi sinonimizada a $C$. breviflora var. pohliana (Windler \& Skinner 1981), por apresentar cálice maior que $15,1 \mathrm{~mm}$ compr. e estípula bem desenvolvida. Neste trabalho, $C$. subdecurrens é reconhecida como uma espécie distinta de C. breviflora, da qual difere pela flor com cerca de 17-22 mm de comprimento, cálice com 17-21 mm de comprimento, ápice do botão floral rostrado e indumento denso-seríceo ou velutino. Em C. breviflora, a flor e o cálice possuem de 10-15(-17) mm de comprimento, ápice floral não rostrado e indumento seríceo ou finamente pubescente.

\section{Agradecimentos}

As autoras agradecem aos curadores dos herbários consultados, à Capes pela bolsa de mestrado concedida para A. M. Filliettaz; à FAPESP pela bolsa de doutorado concedida para A. S. Flores (proc. 00/11674-2).

\section{REFERÊNCIAS BibLIOGRÁfiCAS}

Bentham, G. 1859. Leguminosae. Crotalaria. In Martius, C. F. P. von; Eichler, A. W. $\&$ Urban, I. Flora brasiliensis. F. Fleischer, Lipsiae, 15(1): 18-32.
Bisby, F. A. \& Polhill, R. M. 1973. The role of taximetrics in angiosperm taxonomy II. Parallel taximetric and orthodox studies in Crotalaria L. New Phytologist 72: 727-742.

Filliettaz, A. M. 2002. Estudos taxonômicos de espécies de Crotalaria sect. Calycinae Wight \& Arn. (Leguminosae-PapilionoideaeCrotalarieae) no Brasil. Dissertação de mestrado, Universidade Estadual de Campinas, Campinas.

Flores, A. S. 2004. Taxonomia, números cromossômicos e química das espécies de Crotalaria L. (Leguminosae, Papilionoideae) no Brasil. Tese de doutorado, Universidade Estadual de Campinas, Campinas.

Holmgren, P. K.; Holmgren, N. H. \& Barnett, L.C. (eds.). 1990. Index Herbariorum Part I: The Herbaria of the World. New York Botanical Garden, New York.

Planchuelo, A. M. 1998. Nota sobre el grupo Crotalaria martiana Benth. (LeguminosaeCrotalarieae). Candollea 53: 462-465.

Polhill, R. M. 1968. Miscellaneus notes on African species of Crotalaria. II. Kew Bulletin 22: 169-348.

1981. Crotalarieae. In Advances in Legume Systematics Part 1 (R. M. Polhill \& P. H. Raven, eds.). The Royal Botanic Gardens, Kew, part 1, p. 399-402.

Rafinesque, C. S. 1836. Crotalaria. New Flora and Botany of North America I. p.53-58.

Windler D. R. 1971. New North American unifoliolate Crotalaria taxa (Leguminosae). Phytologia 21: 257-266.

Windler, D. R. \& Skinner, S. G. 1981.Variation in the Crotalaria breviflora complex in Brasil (Fabaceae). Phytologia 49: 425-429. 1982a. New taxa and New Combinations in the American Crotalarias (Fabaceae). Phytologia 50: 185-206. 1982b. The taxonomy and nomenclature of Crotalaria foliosa (Leguminosae) and related species. Brittonia 34: 340-345. 
